\title{
L'intifida d'Al-Aqsa : quelle place pour les citoyens arabes dans l'Etat juif?
}

\section{Laurence Louer}

\section{(2) OpenEdition}

1 Journals

\section{Édition électronique}

URL : http://journals.openedition.org/conflits/397

DOI : $10.4000 /$ conflits.397

ISSN : $1777-5345$

Éditeur :

CCLS - Centre d'études sur les conflits lilberté et sécurité, L'Harmattan

Édition imprimée

Date de publication : 4 mars 2001

ISBN : 2-7475-0725-4

ISSN : 1157-996X

\section{Référence électronique}

Laurence Louer, «L'intifida d'Al-Aqsa : quelle place pour les citoyens arabes dans l'Etat juif ? », Cultures \& Conflits [En ligne], 41 I printemps 2001, mis en ligne le 13 mars 2006, consulté le 30 mars 2021.

URL : http://journals.openedition.org/conflits/397 ; DOI : https://doi.org/10.4000/conflits.397

Ce document a été généré automatiquement le 30 mars 2021.

Creative Commons License 


\title{
L'intifida d'Al-Aqsa : quelle place pour les citoyens arabes dans l'Etat juif?
}

\author{
Laurence Louer
}

1 L'intifada d'al-Aqsa : quelle place pour les citoyens arabes dans l'Etat juif? Laurence LOUËR

2 Au début du mois d'octobre 2000, les citoyens arabes d'Israël ont, pour la première fois dit-on, participé à la lutte de leurs « frères palestiniens » et mené une véritable intifada au cœur même du territoire israélien, offrant douze «martyrs » à la cause palestinienne. Ceux qu'on appelle souvent les «Arabes israéliens » sont à l'origine les Palestiniens qui, au moment de la proclamation de l'Etat israélien en 1948, se trouvaient dans ses frontières ${ }^{1}$ et, en vertu d'un droit du sol qui ne s'applique qu'à eux ${ }^{2}$, sont devenus citoyens israéliens. Réduits à l'état de minorité dans une société faite par et pour les Juifs, les citoyens arabes se sont en réalité trouvés insérés dans une structure sociale caractéristique de ce que Michel G. Smith nomme les sociétés plurales ${ }^{3}$. Ces sociétés sont en général constituées de la réunion forcée de plusieurs groupes ethniques au sein d'un même Etat. Ces derniers vivent chacun dans des domaines sociaux et géographiques strictement ségrégués et se côtoient sans se mélanger dans l'espace économique, voire dans l'espace politique lorsqu'il est pluraliste. L'intégration sociale globale est alors superficiellement réalisée par divers mécanismes, allant de la coercition pure et simple à l'établissement de liens de clientèle entre un groupe dominant qui monopolise l'appareil d'Etat et un ou plusieurs groupes dominés. Dans le cas israélien, la population arabe était ainsi à la fois placée sous étroite surveillance militaire ${ }^{4}$ et reliée au pouvoir central par un réseau de relations unissant les chefs de ses grands clans ruraux aux partis politiques de gouvernement, en particulier le Parti travailliste, omnipotent jusqu'en $1977^{5}$. En outre, diverses agences avaient également développé des stratégies de cooptation des nouvelles élites éduquées dans le système d'éducation moderne ${ }^{6}$. La Histadrout ${ }^{7}$ en particulier, la tentaculaire organisation des travailleurs qui disposait d'un réseau d'entreprises diverses, a 
longtemps constitué l'un des principaux pourvoyeurs d'emplois de cols blancs pour les jeunes diplômés arabes. Système social où la ségrégation est le principal ressort de la domination, la structure plurale de la société israélienne a été au fondement de l'étonnant quiétisme dont ses citoyens arabes ont fait preuve pendant longtemps, source d'une totale marginalisation dans le mouvement national palestinien ${ }^{8}$. Cependant, la violence inédite des événements de l'intifada d'al-Aqsa a précisément été interprétée comme la manifestation extrême des changements qui sont intervenus dans le type de relation qui unit les citoyens arabes à leur Etat depuis les années 70 . L'intifada d'al-Aqsa est en effet venue rappeler que le temps est révolu où la notabilité clanique et les cadres de la Histadrout liés à l'establishment juif dominaient la société arabe d'Israël. Ils ont laissé la place à une nouvelle génération revendiquant sa pleine appartenance au peuple palestinien et entendant jouer un rôle à part entière dans sa lutte de libération. L'action politique ne se résume désormais plus aux échanges clientélistes de services matériels contre des stocks de voix aux partis juifs. En effet, un leadership politique arabe s'est peu à peu constitué au milieu des années $80^{9}$, qui recueille aujourd'hui $70 \%$ du vote arabe et est par là même représenté à la Knesset ${ }^{10}$. Son message repose sur l'identification au peuple palestinien et le soutien à ses objectifs à court terme, en particulier l'établissement d'un Etat palestinien avec Jérusalem Est pour capitale. Il réclame en outre la transformation de l'Etat israélien en "Etat de tous ses citoyens ", c'est-à-dire, en réalité, l'abolition de son caractère juif, considérée comme le seul moyen de parvenir à une égalité complète entre citoyens israéliens juifs et arabes. Renforcé par la large couverture médiatique des discriminations diverses que les citoyens arabes affrontent quotidiennement ${ }^{11}$, ce discours aujourd'hui dominant tient plus généralement lieu de version littéraire ${ }^{12} \mathrm{du}$ statut de victime des citoyens arabes dans la société israélienne et, par là même, fonctionne comme légitimation d'une mobilisation populaire grandissante ${ }^{13}$. Dernier épisode en date de la mobilisation des citoyens arabes de l'Etat juif, l'intifada d'al-Aqsa marque incontestablement une remise en cause des mécanismes quiétistes inhérents aux structures de la société plurale et l'enjeu de l'analyse est d'en évaluer la portée, à la fois pour la communauté arabe et pour la société israélienne tout entière. Les événements et leurs acteurs Etalés sur une dizaine de jours entre le 1er et le 9 octobre 2000 , les affrontements entre la police et les chabab ${ }^{14}$ ont provoqué la mort de douze ${ }^{15}$ citoyens arabes et fait des dizaines de blessés. Ils ont débuté par une grève générale décrétée par le Comité de suivi, l'organe qui fédère l'ensemble du leadership arabe en Israël et souhaitait alors exprimer sa protestation contre la mort des manifestants palestiniens, tués par l'armée israélienne le jour précédent sur l'esplanade des mosquées à Jérusalem. Il faut d'emblée souligner que ce genre de manifestations est très courant dans la population arabe d'Israël: outre les mobilisations portant la revendication de l'égalité complète entre citoyens juifs et arabes, l'expression populaire de soutien au peuple palestinien à diverses phases critiques de son histoire Sabra et Chatila ou la première intifada par exemple - est systématique. Le rapport entre la fréquence de la mobilisation populaire et la fréquence des incidents violents est faible: après avoir été réprimée jusqu'aux années 70, la protestation arabe est aujourd'hui acceptée par les autorités israéliennes et, par là même, routinisée, dans un schéma proche de celui dégagé par Georges Lavau ${ }^{16}$ pour le Parti communiste français. Le leadership nationaliste, institutionnalisé par sa présence à la Knesset, est ainsi cantonné dans une fonction tribunitienne d'entretien plus que de remise en cause du système : en donnant à la protestation une forme ordonnée et un débouché légal, il en 
garantit en réalité le fonctionnement sans explosion. Les événements de l'intifada d'alAqsa dans la population arabe d'Israël ont donc débuté comme une manifestation habituelle, différant seulement peut-être par l'ampleur de la mobilisation, toutes les localités arabes, de la Galilée au nord, au Néguev au sud, ayant répondu à l'appel. La protestation rituelle organisée par le leadership institutionnalisé est cependant cette fois-ci sortie de son cadre tribunitien : suivant en cela la technique de l'intifada dans les territoires palestiniens, des jeunes ont élaboré des barrages de pneus et de poubelles enflammés, bloquant ainsi les grands axes routiers alentour et lançant des pierres aux forces de l'ordre stationnées à l'entrée de leurs villages. Dans les villes, en particulier à Nazareth et Umm al-Fahm, les chabab s'en sont pris aux symboles de l'Etat israélien : banques, bureaux de poste, stations essence et même le Burger King de Nazareth ont été assaillis et parfois tout simplement incendiés. Cagoulés et torses nus, les jeunes ont, six jours durant, défié la police, dans ce qu'ils nomment eux-mêmes un «jeu » qui a rapidement dégénéré, les forces de l'ordre répliquant avec des balles, caoutchoutées ou réelles. L'épilogue tragique de ces affrontements meurtriers est venu dans la nuit du 9 octobre. Descendus de Natzéret Illit - la ville construite dans les années 60 dans le cadre du plan de judaïsation de la Galilée et qui surplombe aujourd'hui la ville arabe de Nazareth -, un groupe de Juifs excédés, sentant leur sécurité directement menacée par l'émergence de ce que la presse israélienne a largement qualifié de «second front » de la « guerre » qui oppose toujours Israël et les Palestiniens, a attaqué le Quartier Est de Nazareth. Deux nouveaux « martyrs » arabes sont alors venus s'ajouter aux dix autres. Les circonstances obscures de leur mort ont alimenté la polémique sur l'attitude partiale de la police. Les Arabes l'accusent en effet d'avoir pris parti pour les Juifs agresseurs et tiré à balles réelles sur la foule des habitants qui s'étaient rassemblés pour défendre leur quartier ${ }^{17}$. On l'a compris, les acteurs de cette intifada des Arabes de $48^{18}$ sont, comme dans les territoires palestiniens, les chabab, les jeunes hommes. A l'exception de l'une des deux victimes des affrontements avec les civils juifs à Nazareth, les martyrs ont tous entre 18 et 26 ans. On n'y trouve pas d'enfants, dont la place, chez les citoyens arabes, reste à la maison et non pas dans la rue, contrairement à la situation qui prévaut en Palestine. Ils sont en outre tous musulmans, élément important à souligner quand on sait que huit des douze martyrs sont tombés dans des localités mixtes où coexistent chrétiens et musulmans ${ }^{19}$. De même, la liste des 109 personnes arrêtées établie par l'association Mossawa ne fait apparaitre aucun nom chrétien ${ }^{20}$. Les Druzes, quant à eux, ont naturellement persisté dans leur stratégie communautaire propre, basée sur une collaboration totale avec l'Etat hébreu dont l'élément central est l'acceptation de la conscription obligatoire, en échange de laquelle ils ont obtenu un statut officiel séparé de celui des autres Arabes ${ }^{21}$. S'ils ont participé aux événements, c'est de l'autre côté, parmi les forces de l'ordre chargées de la répression, dans une moindre mesure, il est vrai, en Israël que dans les territoires palestiniens où plusieurs d'entre eux ont été blessés ${ }^{22}$. L'homogénéité confessionnelle des chabab tendrait à confirmer l'hypothèse de l'islamisation du conflit israélopalestinien et, corollairement, de la communauté arabe d'Israël. D'autres arguments accréditent cette thèse, comme par exemple l'omniprésence de la symbolique islamique et les motivations exprimées par les chabab, affirmant que, par leur action, ils entendaient participer au combat pour la défense d'al-Aqsa. Il faut ajouter à cela l'omniprésence des leaders du Mouvement islamique, l'organisation qui se réclame des Frères musulmans et qui est aujourd'hui devenue le premier mouvement politique chez les citoyens arabes. L'idée de l'islamisation doit cependant être nuancée par le fait que 
la mobilisation inédite des Arabes de 48 a soulevé l'enthousiasme général des milieux nationalistes divers qui, Chrétiens et Musulmans confondus, ont préféré voir dans alAqsa un symbole national transconfessionnel, incarnant la question de Jérusalem plus que celle de la défense de l'islam. Les divers courants nationalistes ont ainsi eu à cœur de ne pas laisser le monopole de la symbolique d'al-Aqsa au Mouvement islamique. Comme à son habitude, ce dernier a d'ailleurs lui-même joué le jeu de l'unité nationale. Ainsi, dans son bastion galiléen de Kufr Kanna qui comprend une importante population chrétienne, le cheikh Kamal Khatib s'est empressé de souligner que les Chrétiens avaient activement participé à la mobilisation pour al-Aqsa et apporté une généreuse contribution financière au comité chargé de l'aide aux familles des martyrs. Enfin, pour écarter les thèses classiques du complot et des classes dangereuses, il faut ajouter que les douze martyrs, groupe générationnellement et confessionnellement homogène, est hétérogène quant aux opinions politiques et aux occupations professionnelles. Les trois courants politiques présents à la Knesset sont ainsi représentés. Quant aux statuts socioprofessionnels, on trouve également un large spectre, allant de l'étudiant brillant venant d'obtenir une bourse d'études pour les Etats-Unis, au jeune travailleur du marché aux légumes de Tel Aviv, à l'ingénieur récemment diplômé, en passant par l'animateur d'un club sportif. Les martyrs ne sont donc ni des activistes politiques ayant eu un rôle organisationnel, ni des marginaux naturellement portés à la violence, ainsi que l'ont pourtant suggéré les services de sécurité en mettant en cause le parti d'Azmi Bishara, le Rassemblement patriotique démocratique, et en affirmant avoir constaté la présence active de « criminels parfois armés ${ }^{23}$. Comme le souligne Antony Oberschall ${ }^{24}$, ces deux thèses du complot et des criminels sont typiques de l'attitude des autorités qui, par là, visent à dépolitiser les mouvements populaires en niant toute idée d'un lien organique entre les acteurs mobilisés et la population dont ils se réclament. Cependant, dans le contexte d'une société plurale ainsi divisée en deux groupes ethniques dont les intérêts apparaissent de plus en plus divergents, le fait que ce type d'interprétation ait prévalu dans les services de sécurité contrebalance les risques de réactions radicales d'une partie de la population juive. En effet, les affrontements entre civils juifs et arabes à Nazareth le 9 octobre ont été suivis par d'autres du même type dans les jours suivants. En général dans les villes mixtes où Juifs et Arabes cohabitent, des petits groupes de civils juifs s'en sont pris aux biens et aux personnes arabes, ajoutant encore au désarroi d'une opinion publique qui s'est alors mise à évoquer l'éventualité d'une "guerre civile». C'est dans ce contexte que l'idée du transfert des citoyens arabes hors de l'Etat israélien a refait surface : un sondage du quotidien Maariv indiquait en effet que $60 \%$ des personnes interrogées y étaient favorables ${ }^{25}$. Face à l'ambiance de panique qui a ainsi pu saisir l'opinion israélienne, le Shin Bet $^{26}$ a finalement adopté une position intégratrice, choisissant la thèse du complot et des classes dangereuses mais mettant également en cause les politiques des gouvernements successifs vis-à-vis des Arabes, qu'il a qualifiées de "faibles et insuffisantes $~^{27}$. Il a en outre formulé quelques propositions concrètes destinées à remédier au sentiment de discrimination des citoyens arabes, allant jusqu'à préconiser, outre des moyens budgétaires appropriés et l'intégration dans la direction des grandes entreprises publiques, des mesures symboliques, comme par exemple la nomination d'un ministre arabe. Une structure d'épuration ethnique? La configuration inédite des événements de l'intifada d'al-Aqsa dans la communauté arabe d'Israël amène donc à s'interroger sur ses suites potentiellement radicales. Pour cela, il est utile de passer d'une analyse macro à une analyse micro, en se penchant sur les relations 
interethniques individuelles entre Juifs et Arabes. Les universités israéliennes constituent un bon site d'observation, en particulier pour appréhender ces relations interethniques dans leur rapport aux mécanismes de la protestation arabe en Israël. En effet, elles ont connu cette année une vague d'agitation organisée par le leadership étudiant arabe qui, a posteriori, a été interprétée comme un signe annonciateur de l'intifada. Les manifestations, qui se sont principalement déroulées à l'université de Haïfa qui compte le plus grand nombre d'étudiants arabes (20\%), avaient des motifs divers. Les premières entendaient protester contre la discrimination entre Arabes et Juifs à l'université. Les autorités de l'université ayant interdit les manifestations, les étudiants s'empressèrent de les maintenir et d'en organiser d'autres pour protester, cette foi, contre les manquements au principe de la liberté d'expression. En avril, enfin, les manifestations prirent un tour violent quand les étudiants entreprirent d'exprimer leur colère face aux événements du Jour de la terre. Célébré tous les 30 mars depuis 1976, ce temps fort des rituels de protestation arabes commémore la mort de sept personnes qui participaient à une manifestation contre la vague d'expropriation de terres qui a touché la population arabe dans les années 70. Depuis longtemps transformé en pique-nique familial, il a dégénéré cette année 2000 lorsqu'une cinquantaine de chabab ont attaqué à coups de pierre une base militaire située à l'entrée de la ville de Sakhnine en Galilée. Une vieille femme, à laquelle fût aussitôt décerné le titre de "martyre ", décéda quelques jours plus tard, intoxiquée, d'après les Arabes, par les gaz lacrymogènes lancés par la police. Suite à la manifestation étudiante, la police pénétra pour la première fois dans l'enceinte de l'université de Haïfa pour disperser les manifestants qui brandissaient des drapeaux palestiniens. S'ensuivit une autre vague de manifestations pour protester contre la répression et la mobilisation étudiante ne prit fin qu'avec l'arrêt des cours en juin. Dans un tel contexte, on pourrait penser que la vie étudiante dans les universités israéliennes s'est transformée en espace de conflit interethnique. Il n'en est pas tout à fait ainsi cependant. En effet, aussi bien au travers de l'observation participante que par le recueil d'entretiens biographiques ${ }^{28}$, on trouve que la caractéristique principale des relations interpersonnelles entre Arabes et Juifs dans le cadre universitaire est le maintien et non la rupture des mécanismes de la reproduction sociale. Dans la lignée des travaux d'Erving Goffman, on constate avant tout un soucis, chez la majorité des étudiants arabes, de tout faire pour réduire la tension dans leurs relations avec les Juifs. $\mathrm{Au}$ quotidien, différentes formes de tact sont ainsi mises en œuvre, dont la principale est l'interdit d'aborder les questions politiques avec les Juifs. Beaucoup décrivent ainsi leur préoccupation permanente de maintenir des relations amicales avec leurs collègues juifs en évitant de ramener systématiquement les relations interpersonnelles à des relations entre deux entités collectives en conflit, les Arabes et les Juifs. Les relations routinières se déroulant dans certains secteurs spatio-temporels - les salles de cours, la bibliothèque, la cafétéria ou encore la cité universitaire - sont considérées comme devant rester hors de l'espace-temps du conflit. Cependant, ces mêmes étudiants qui affirment leur souci constant de réparer les tensions ont également presque tous participé aux manifestations, ce qui constitue en revanche un acte délibéré de création de tension. On constate ainsi que les étudiants ne sont pas divisés entre une majorité de quiétistes pratiquant l'évitement et une minorité d'agitateurs créant délibérément de la tension au lieu de tenter de la réparer. En réalité, le phénomène de la protestation étudiante répond à une logique de sectorisation : il y a des espaces-temps légitimes et illégitimes pour la protestation. Il n'est ainsi pas 
question de faire irruption dans les salles de cours et d'inciter les étudiants à quitter les rangs pour participer à une manifestation. Peu d'étudiants acceptent en outre de manquer un cours pour aller manifester: ils ne le font que s'ils ont du temps libre. Enfin, les manifestations se déroulent toujours dans des lieux précis du campus universitaire, principalement dans les espaces verts situés à l'extérieur des bâtiments. Même les activistes politiques le reconnaissent : leur objectif est de négocier avec la direction de l'université un espace-temps légitime de protestation au sein du campus et non d'organiser une protestation disruptive étendue à tous les secteurs de la vie étudiante. Après les événements de l'intifada, les leaders étudiants ont d'ailleurs reçu des consignes strictes de la part de la direction nationale des partis auxquels ils sont affiliés : il leur fallait continuer la mobilisation mais se cantonner aux espaces-temps légitimes qui devaient, autant que possible, être négociés avec les autorités universitaires. Dans un contexte particulièrement tendu, la réaffirmation du principe de sectorisation de la protestation leur apparaissait comme une garantie contre une rupture des mécanismes profonds de la reproduction sociale qui peut caractériser certaines situations d'épuration ethnique. La police, nouvel acteur du conflit La protestation arabe en Israël est donc à la fois routinisée car canalisée par un leadership nationaliste intégré au système dans une fonction tribunitienne, et sectorisée dans une logique non-disruptive de la reproduction sociale. Si l'intifada d'al-Aqsa marque une rupture dans ces deux dynamiques, c'est probablement dans le rôle inédit qu'y a joué la police. Dans une telle configuration de la protestation en effet, les affrontements violents entre la police et les manifestants sont rares. Plus encore, se comportant comme un Etat typique des sociétés plurales, Israël a opté dès le début pour une gestion indirecte de ses citoyens arabes passant autant que possible par des intermédiaires issus de la communauté elle-même. Le policier comme agent de répression violente ne fait dès lors pas partie de l'imaginaire de la population. Dans le récit identitaire arabe, basé sur l'entretien du statut de victime, le personnage honni incarnant le contrôle de l'Etat est l'agent de la Shabak ${ }^{29}$, le «mousta'rib ", littéralement l'espion «arabisé », qui infiltre d'autant mieux la société arabe qu'il est en général de culture arabomusulmane. De fait, la gestion sécuritaire des Arabes est essentiellement aux mains du Shin Bet et non de la police. Dès lors, non seulement les affrontements avec les forces de l'ordre sont rares, surtout comparés à la fréquence des manifestations populaires, mais la police est quasiment inexistante dans les localités ethniquement homogènes où vivent la plupart des Arabes ${ }^{30}$. Outre le réseau des services secrets, cette absence est également compensée par le succès des Unités de défense civile, une formule qui repose sur le volontariat d'habitants qui assurent la sécurité ordinaire de leur village et ne sont pas considérés par leurs compatriotes comme des éléments allogènes à l'ordre local. Les ruptures de l'ordre public sortant de l'ordinaire, en particulier les crimes de sang, sont gérées conjointement par la police et par les comités de conciliation. Ces institutions traditionnelles d'autorégulation des conflits font signer aux parties un accord qui sera ensuite pris en compte par le tribunal. Quel a donc été, dans ce contexte, le moteur de cette nouvelle interaction explosive entre les chabab et la police? Dans un premier temps, on peut considérer que l'intifada d'al-Aqsa a brisé le principe de sectorisation. Ainsi, le blocage des axes routiers a-t-il été explicitement mis en avant par Ehoud Barak comme inacceptable et justifiant, à lui seul, l'intensité de la réplique policière : l'irruption de la protestation arabe dans l'espace géographique de la société globale à un moment critique de son histoire a ainsi été présentée comme l'explication-clef de l'extra-ordinarité des événements. Il est vrai que, en raison même 
des structures ségrégantes propres aux sociétés plurales, l'un des aspects de la sectorisation de la protestation arabe réside dans la circonscription spatiale de la plupart des défilés, qui se déroulent, à quelques exceptions près ${ }^{31}$, dans les localités arabes et passent inaperçus de la population juive. Cependant, on peut opposer à cet argument que si la mortalité de l'intifada d'al-Aqsa est inédite, le blocage de routes, les jets de pierres et de cocktails Molotov, et la destruction des bâtiments publics ne le sont pas. En effet, au début de la première intifada, les citoyens arabes avaient naturellement manifesté leur soutien au peuple palestinien en bloquant des axes routiers importants et en jetant pierres et autres cocktails Molotov. La manifestation avait finalement été dispersée par des moyens traditionnels non-létaux. A cette occasion, Elie Rekhess avait parlé d'une violence récurrente - et donc prévisible - en réaction aux événements du conflit israélo-palestinien ${ }^{32}$. Par la suite, plusieurs journées d'émeutes ont marqué les localités arabes d'Israël durant l'intifada, en particulier en mai 1990 suite à l'assassinat de sept Palestiniens par un Israélien ${ }^{33}$. Doit-on alors penser que l'intifada d'al-Aqsa se singularise simplement par une différence de degré, degré dans l'intensité de la violence des chabab et, conséquemment, de la police ? Cette hypothèse de la radicalisation a bien sûr été soutenue par les forces de l'ordre. Outre qu'elle contredit les deux principes de la fonction tribunitienne et de la sectorisation dégagés plus haut, elle occulte l'aspect fondamentalement interactif des mobilisations populaires. Comme le souligne Antony Oberschall, « c'est la réaction des autorités, plus que les actions et les plans de l'opposition, qui détermine le cours subséquent des événements, et en particulier si le conflit reste pacifique ou devient violent $\aleph^{34}$. En l'occurrence, la gestion des précédents de la première intifada montre que c'est sans doute la perception des autorités, plus que l'attitude de la population arabe, qui a changé et c'est ce qu'il s'agit d'expliquer. Pour cela, il est indispensable de rappeler la gestion de la crise actuelle dans les territoires palestiniens, qui offre en effet un parallèle saisissant : c'est bien la réaction de l'armée israélienne, choisissant d'ouvrir le feu sur les manifestants de l'esplanade des mosquées, qui a transformé une simple émeute en nouvelle intifada. L'intensité de la réaction en Cisjordanie et à Gaza a conditionné l'intensité de la réaction en Israël : les manifestations de solidarité des citoyens arabes avec leurs "frères" palestiniens ont été vécues comme l'ouverture d'un second front et non comme un exemple de plus de la protestation routinisée et sectorisée. Les motifs de ce changement dans la perception israélienne du conflit israélo-palestinien restent encore largement sujets à controverse et demanderaient une analyse à eux seuls. Au-delà des explications psychologisantes mettant en avant la surprise et le sentiment de trahison vis-à-vis du partenaire palestinien, sans doute fautil aussi y voir les effets des enjeux de pouvoir intérieurs dans le contexte d'une grave crise gouvernementale. A cet égard, les divergences d'appréciation entre le Shin Bet et l'armée, le premier souhaitant éviter une rupture totale avec les Palestiniens, la seconde poussant au contraire à plus de répression ${ }^{35}$, pourraient sans doute constituer un indice pour interpréter la gestion sécuritaire de l'intifada des Arabes de 48. On a vu en effet que le Shin Bet, en raison même de son excellente connaissance du terrain, avait fait preuve de modération dans son appréciation de la crise et des solutions à lui apporter. Par contraste, le discours policier, reflétant à son tour bien la position d'extériorité totale de la police par rapport à la société arabe, appelait à plus de fermeté, soulignant la nécessité de renforcer la présence policière dans les villages et de modifier ainsi le mode de gestion indirecte adopté jusqu'à présent ${ }^{36}$. Prise dans sa globalité, la gestion sécuritaire de la crise dans les territoires palestiniens et en Israël 
reflète finalement bien la bipolarisation de la société et du champ politique israéliens quant aux solutions préconisées pour mettre fin au conflit israélo-palestinien. Au-delà des différences touchant à la connaissance du terrain, les divergences d'appréciation entre le Shin Bet d'un côté, et la police et l'armée de l'autre, reflètent aussi la lutte entre le camp des faucons et celui des colombes, qui se manifeste à presque tous les niveaux de décision. Rupture et réconciliation Quelles que soient les raisons de la perte de confiance entre Israéliens et Palestiniens, l'idée que l'intifada d'al-Aqsa représente un tournant dans les relations entre citoyens israéliens arabes et juifs a constitué le discours dominant. Des deux côtés, il s'agissait entre autres de réfuter la thèse du mouvement pour les droits civiques. «Les Arabes n'ont pas déclenché une révolte pour obtenir plus de budgets ou l'égalité civile [...] Les Arabes d'Israël [...] ne veulent pas de nous ici. Ils veulent leurs terres, sur lesquelles des centaines de kibboutz, de mochav ${ }^{37}$, de petites communautés et même des villes ont été bâtis ", affirmait ainsi une tribune libre de $\mathrm{Ha}^{\prime} \operatorname{aretz}^{38}$. En qualifiant d'emblée les événements d' «intifada », les Arabes ont également alimenté l'idée que leur mobilisation faisait partie intégrante de la lutte nationale du peuple palestinien pour la libération de Jérusalem: la violence aurait précisément été un moyen d'exprimer que «la protestation est d'abord une protestation politique et seulement ensuite une protestation contre la situation socioéconomique et les conditions de vie générales pitoyables ${ }^{39}$. L'intellectuel islamiste Mohannad Moustapha a clairement résumé l'enjeu des événements tel que l'a appréhendé la majorité du leadership. Insistant sur la nécessité de saisir l'occasion historique de rompre le partenariat de fait entre la communauté arabe et le camp de la paix, il refuse la légitimation offerte par une partie de la gauche: "la gauche israélienne essaye de dire quelque chose de très dangereux: "s'il y avait l'égalité, pourquoi la minorité arabe d'Israël aurait-elle fait l'intifada ?" [...] On tente aujourd'hui d'effacer les dimensions religieuses et patriotiques de l'intifada d'al-Aqsa et de la transformer en question essentiellement civile. Encore une fois, la majorité oppresseuse et ses institutions travaillent à justifier le comportement de la minorité en invoquant la difficulté des circonstances. Or, cela est encore pire que lesdites circonstances difficiles dont ils parlent $\aleph^{40}$. Chez les Arabes, le discours du tournant reposait donc sur la recherche d'une alternative à l'alliance officieuse qui lie depuis maintenant une quinzaine d'années les partis arabes et le Parti travailliste. En effet, le leadership nationaliste aujourd'hui intégré au système parlementaire après en avoir été systématiquement écarté est né à la faveur de la bipolarisation de l'espace politique israélien au milieu des années 80 . Alors dans l'incapacité de faire la différence avec le Likoud, le Parti travailliste s'est lancé dans une stratégie de mobilisation maximale des soutiens, cooptant, entre autres, une partie des élites arabes nationalistes. Ces dernières, parfois ouvertement soutenues par l'OLP, ont ainsi pénétré la Knesset, canalisant un vote protestataire grandissant tout en le transmutant en soutien à l'action parlementaire des travaillistes auxquels ils apportaient des voix alors cruellement manquantes ${ }^{41}$. Finalement clairement constitué en "camp de la paix " après la signature des accords d'Oslo, le Parti travailliste a alors vu une transformation qualitative de son implantation dans la population arabe. En raison de son passé de parti dominant jusqu'en 1977, il avait pu maintenir un vaste réseau de clientèle, passant en particulier par la section arabe de la Histadrout. Aujourd'hui nettement amoindri, ce réseau assure au parti moins de voix que l'idéologie pacifique véhiculée par ses colombes qui, en 1996 et 1999, a poussé 95 \% des électeurs arabes à voter pour le candidat travailliste à la présidence du gouvernement. La gauche non-travailliste étant 
restée trop longtemps murée dans un silence consterné à leur goût, et Ehoud Barak ayant perdu son image de faiseur de paix, on comprend bien que le leadership et la population arabes dans son ensemble aient souhaité trouver une alternative au partenariat avec les travaillistes, quitte à aller jusqu'au boycott. Cependant, ces tentatives se sont révélées vaines et, finalement, une fois la date des élections avancée, la force intégrative du système politique israélien s'est à nouveau manifestée, peut-être plus que jamais. En effet, le Front démocratique pour la paix et l'égalité a d'emblée annoncé son intention de soutenir un candidat de la paix alternatif à Ehoud Barak, proposant à la gauche non-travailliste la formation d'une troisième voie. Le Rassemblement patriotique démocratique, qui a toujours basé son identité politique sur son homogénéité ethnique par opposition à son rival direct, à l'idéologie nationaliste tout aussi marquée mais formellement judéo-arabe ${ }^{42}$, a quant à lui annoncé son intention de se transformer en parti judéo-arabe, ouvrant ses rangs à tous les faiseurs de paix. Les partis arabes dans leur ensemble ne se sont finalement résolus à appeler au boycott des élections qu'une fois la candidature de Shimon Pérès avortée, laissant toutefois la porte ouverte à un revirement si Ehoud Barak parvenait à un accord de paix satisfaisant avec les Palestiniens d'ici la tenue des élections. En choisissant de concentrer ses critiques sur la personne d'Ehoud Barak, le leadership arabe n'est finalement pas parvenu à penser une stratégie politique alternative au partenariat avec la gauche. La logique d'intégration politique apparaît d'autant plus incontournable qu'aucune nouvelle direction politique n'a, pour le moment, émergé de l'intifada d'alAqsa. Dans les configurations où une élite politique institutionnalisée médiatise la protestation, ce genre de mobilisation est pourtant souvent l'occasion où l'on voit apparaître un leadership radical, critiquant la collusion de la première avec le pouvoir oppresseur et préconisant la rupture totale. A cet égard, l'hypothèse d'un Mouvement islamique subissant une transformation qualitative à l'instar des Frères musulmans palestiniens, passés, en prenant le nom de Hamas, d'une stratégie quiétiste d'islamisation par le bas à une stratégie radicale, si elle n'est pas à exclure totalement, apparaît peu probable. En effet, s'il est vrai qu'une partie du Mouvement islamique refuse la participation aux élections nationales ${ }^{43}$ au motif qu'elle équivaut à une instrumentalisation ne débouchant sur aucun effet réellement bénéfique, la stratégie qu'il préconise s'apparente plus au repli communautaire qu'à l'action violente. Ainsi, outre le travail d'islamisation des mœurs, le mouvement, qui dirige plusieurs conseils locaux, incite la population à se prendre elle-même en charge sans attendre les hypothétiques fonds gouvernementaux. Sa logique repose sur l'autonomie de la communauté musulmane: la population est invitée à financer elle-même certaines infrastructures, que ce soit le réseau de canalisation, le goudronnage des rues ou les terrains de sport. Cette stratégie n'exclut d'ailleurs pas le partenariat avec l'Etat: beaucoup d'imams proches de cette branche du Mouvement islamique sont ainsi fonctionnaires du ministère des Religions. Conflits interethniques et déségrégation Pour conclure, il est utile de revenir sur la mobilisation étudiante de l'université de Haïfa, dont l'épilogue est encore une fois riche d'enseignement. Peu avant la rentrée universitaire prévue en novembre, la direction de l'université ainsi que les leaders étudiants prévoyaient une intensification de la mobilisation arabe en réaction aux événements de l'intifada. En prévision des tensions, les effectifs de sécurité avaient été augmentés. Cependant, au fur et à mesure que les semaines passaient, force fut de se rendre à l'évidence que les violences annoncées n'avaient pas eu lieu. Deux mois après la reprise des cours, seules deux manifestations d'étudiants arabes avaient été 
organisées, sans qu'aucun incident eut été à déplorer. Les raisons avancées par la direction et les organisations étudiantes pour expliquer le décalage entre les expectatives et la réalité des faits sont significatives. D'une part, les manifestations ont été annoncées à l'avance et n'ont pas été interdites par la direction de l'université. D'autre part, elles se déroulent dans des endroits plus éloignés du centre du campus que l'année passée. Enfin, les leaders étudiants soulignent que le directeur, qui est nouvellement entré en fonction cette année, est en contact régulier - et cordial - avec eux. L'évolution de la mobilisation étudiante, qui n'a pas suivi le cours prévu, montre encore une fois que l'enjeu est bien la négociation d'espace-temps légitimes de la protestation au sein de la société globale. Il s'agit de rompre le lien qui existe jusqu'à maintenant entre la sectorisation de la mobilisation et la ségrégation de l'espace géographique. Dans le cadre d'une telle configuration en effet, la protestation ne touche pas la population juive, s'apparentant de plus en plus à un rituel d'entretien de la solidarité communautaire et de moins en moins à un moyen de pression efficace. Le leadership institutionnalisé, quant à lui, s'il canalise la protestation et constitue une voie de communication avec le pouvoir central, apparait également limité dans sa capacité d'infléchir le cours des politiques publiques vis-à-vis des Arabes. En outre, il n'a qu'un pouvoir de rétribution limité vis-à-vis de ses électeurs. En effet, au contraire d'autres élites communautaires présentes au parlement qui entretiennent leur électorat en lui redistribuant une partie des ressources publiques ${ }^{44}$, les députés arabes étant toujours exclus des coalitions gouvernementales, n'ont rien à redistribuer. La population arabe est bien consciente de la marge d'action restreinte de ses représentants, dont la fonction se résume, là encore, de moins en moins à la médiation des griefs et de plus en plus à la pure représentation théâtrale de l'identité palestinienne. Dans un tel contexte, il apparaît d'autant plus nécessaire de ne pas fermer les canaux de la protestation populaire. Il apparait tout aussi indispensable de mettre fin au sentiment grandissant que le leadership parlementaire arabe est victime d'un jeu de dupe où son soutien au camp de la paix n'a pas de véritables conséquences quant à l'amélioration des conditions matérielles générales de la population arabe. L'entretien de ce sentiment croissant d'instrumentalisation à sens unique du vote arabe ne pourrait que donner raison aux partisans du retrait communautaire, on l'a vu déjà bien organisés au sein du Mouvement islamique. Enfin, reste à souligner que, dans les sociétés plurales, les conflits entre les groupes ethniques surgissent précisément dans les phases de déségrégation. C'est alors que, au travers de nouvelles élites de diverses natures selon les contextes, des groupes marginaux jusque-là invisibles à la société globale parce que cantonnés dans certains espaces sociaux et géographiques, pénètrent de nouveaux domaines. Or, par sa logique de débordement en dehors des cadres géographiques auxquels la protestation arabe se cantonne d'habitude, l'épisode dramatique de l'intifada d'al-Aqsa montre bien que, du point de vue des relations entre Juifs et Arabes, Israël est bien une société en phase de déségrégation. Sur le temps long, la constitution d'une nouvelle élite politique qui pénètre l'espace public israélien en proclamant son identité palestinienne en est également l'une des manifestations. Un autre exemple d'éclatement des frontières qui séparent Juifs et Arabes est la migration interne des Arabes vers de nouveaux centres urbains, où ils constituent progressivement des noyaux de mixité ethnique appelés à se développer ${ }^{45}$. Plus généralement, sous l'effet de l'accès aux études supérieures, les frontières sociales qui cantonnent les Arabes à des activités économiques et des statuts sociaux limités commencent aussi à s'élargir. Une petite et moyenne bourgeoisie se constitue ainsi peu 
à peu autour d'un noyau d'avocats, de comptables ou de médecins, dont la réussite ne doit cependant pas occulter le phénomène de déclassement dont sont victimes les diplômés des universités. Ni le système d'éducation publique en langue arabe, ni une économie arabe faite de petits commerces, ni même un réseau associatif spécialisé dans l'action en justice, ne parviennent en effet à les absorber. Si d'autres secteurs socioprofessionnels ne s'ouvrent pas à eux, ils risquent de se constituer en une élite intellectuelle frustrée de ses ambitions dont le potentiel explosif a déjà été souligné dans d'autres contextes socio-historiques ${ }^{46}$. En ce sens, la déségrégation apparait tout autant un fait qu'une nécessité. Cependant, si la logique de déségrégation est potentiellement conflictuelle, c'est qu'elle ne concerne pas seulement les groupes marginaux mais également le groupe dominant. En effet, en brisant les frontières qui les séparent de la société globale, les premiers provoquent aussi souvent la déstabilisation des catégories les plus fragiles de la population dominante, qui se sentent directement menacées, dans leur statut social ou leur activité professionnelle ${ }^{47}$. Pour le cas d'Israël, le problème de la déségrégation n'est donc pas seulement celui de l'intégration des citoyens arabes mais aussi celui de la réaction de la population juive. Est-elle prête à accepter les conséquences de la pénétration des nouvelles élites arabes dans la société israélienne? Sans parler d'un Etat véritablement consociatif associant Juifs et Arabes à parité en tant que deux entités nationales, une plus grande mixité sociale, associée à un inévitable rééquilibrage démographique en faveur de la population arabe, impliquerait en effet une binationalité de fait. A cet égard, la bipolarisation du champ politique évoquée plus haut se retrouve au niveau des opinions quant à la place des citoyens arabes : l'attitude colombe ou faucon quant au sort des territoires palestiniens est fortement prédictive de l'attitude face à la minorité arabe $^{48}$. Ce lien entre la question palestinienne générale et la minorité arabe citoyenne de l'Etat israélien doit être aujourd'hui considéré dans la perspective de création d'un Etat palestinien. Une fois la question nationale palestinienne institutionnellement résolue, il est en effet peu probable qu'une majorité de la population juive soit prête à soutenir une remise en cause du caractère juif de la société et de l'Etat israéliens.

\section{NOTES}

1. A l'époque, leur nombre s'élevait à 156000 . Les citoyens arabes sont aujourd'hui 1 100000 et représentent $17,5 \%$ de la population israélienne totale.

2. Que l'on peut opposer à un droit du sang pour les Juifs, puisque, dans le cadre de la Loi du retour qui fait de tout Juif un citoyen israélien potentiel, ces derniers obtiennent la citoyenneté israélienne en vertu de leur caractère intrinsèque de Juifs.

3. Voir Leo Kuper and Michael G. Smith, Pluralism in Africa, Los Angeles, University of California Press, 1969.

4. La population arabe a été placée sous administration militaire jusqu'en 1966. A ce sujet, on peut voir le livre de Sabri Jyriès, Les Arabes en Israël 1948-1967, Beyrouth, Centre de recherches palestiniennes, 1969. 
5. Date à laquelle le Likoud prend pour la première fois le pouvoir. Pour une étude détaillée du clientélisme politique et du clanisme, voir le livre d'Abner Cohen, Arab Border Villages in Israel : A Study of Continuity and Change in Social Organization, Manchester, Manchester University Press, 1965.

6. Pour une étude détaillée des mécanismes de cooptation des nouvelles élites, voir le livre de Ian S. Lustick, Arabs in the Jewish State. Israel's Control of a National Minority, Austin, University of Texas Press, 1980.

7. Acronyme hébreu de la Fédération générale du travail, dont le rôle de proto-Etat fut essentiel pour le mouvement sioniste durant la période pré-étatique.

8. Pour la perception des citoyens arabes d'Israël par la résistance palestinienne, voir l'article de Hillel Frisch, «The PLO and the Arabs in Israel 1967-93 : Politicization or Radicalization?", Nationalism \& Ethnic Politics, vol. 2, n 3, autumn 1996.

9. Pour une analyse du processus de constitution de ce nouveau leadership arabe, voir p. 120.

10. Trois listes arabes sont actuellement représentées à la Knesset, qui ont pour ainsi dire toutes le même programme : la Liste arabe unie (Raam) qui compte cinq députés et représente $31 \%$ des voix arabes, la liste mixte du Front démocratique pour la paix et l'égalité (Hadash) avec trois députés - deux arabes et une juive - et 21,3\% du vote arabe, et la liste du Rassemblement uni patriotique (Balad) avec deux députés et 16,7 \% du vote arabe. En outre, quatre députés arabes sont élus sur des listes de partis juifs, deux au Parti travailliste, un au Likoud et un au Meretz (situé à la gauche du Parti travailliste). A cet égard, il faut cependant souligner que deux d'entre eux (au Likoud et au Parti travailliste) sont issus de la communauté religieuse druze dont la situation n'est pas comparable à celle des autres Arabes en Israël. A ce sujet, voir p. 112.

11. Sans parler des journaux arabes, pour ainsi dire spécialisés dans cette tâche, le grand quotidien Ha'aretz propose très souvent à ses lecteurs des comptes-rendus des difficultés socio-économiques propres aux citoyens arabes.

12. Pour reprendre les termes d'Erving Goffman, Stigmate. Les usages sociaux des handicaps, Paris, Les éditions de minuit, 1975, p. 38.

13. Antony Oberschall a bien souligné l'importance de l'existence d'une telle version littéraire des griefs dans les processus de mobilisation. Voir Social Conflict and Social Movements, New Jersey, Englewood Cliffs, 1973, p. 304.

14. Les chabab, littéralement les « jeunes » en arabe, ont été les principaux acteurs de l'intifada dans les territoires palestiniens entre 1987 et 1993 . Ce sont également eux qui sont en première ligne dans les affrontements actuels avec l'armée israélienne.

15. Le chiffre de treize victimes avancé par les médias israéliens prend en compte un jeune Palestinien de la bande de Gaza mort lors des affrontements à Umm al-Fahm, une ville arabe israélienne située en bordure de la Cisjordanie.

16. Georges Lavau, A quoi sert le Parti communiste français ?, Paris, Fayard, 1981.

17. La police dément bien sûr cette version des faits. L'origine des balles reste difficile à établir. La commission d'enquête chargée de faire la lumière sur les événements apportera peut-être une réponse dans les mois à venir.

18. Nom que les citoyens arabes aiment à se donner pour souligner leurs liens avec les Palestiniens des territoires qui, eux, sont alors désignés par l'expression « Arabes de 67 » : les Arabes d'Israël ont été fondés en tant que groupe en 1948, ceux de Cisjordanie et de Gaza en 1967 avec l'occupation israélienne de ces territoires. 
19. Nazareth, Kufr Kanna, Sakhnine et 'Arrabé. D'après les chiffres de mai 2000 donnés par le Bureau central des statistiques, les Chrétiens représentent $10 \%$ de la population arabe d'Israël.

20. Mossawa Center. The Advocacy Center for Arab Palestinian Citizens of Israel : Black October. Israel Slaughters its Own Citizens.

21. Les Druzes constituent $9 \%$ de la population arabe d'Israël. Leur statut séparé implique, entre autres, une législation de statut personnel calquée sur celle des Druzes libanais et distincte de celle des Musulmans, un secteur éducatif propre, ainsi que la mention « Druze » au lieu de " Arabe » sur la carte d'identité. Pour une étude en français récente du destin particulier de la communauté druze d'Israël, voir la thèse d'Isabelle Rivoal, Les Maîtres du secret. L'identité communautaire et ses manifestations au Proche-Orient : le cas des Druzes en Israël, thèse EHESS, Paris, 1997.

22. Un jeune soldat druze est même mort lors de la prise du Caveau de Joseph à Naplouse par les Palestiniens, ce qui a entraîné une polémique dans la communauté druze qui a accusé l'armée israélienne de racisme, estimant qu'elle aurait pu sauver le jeune homme qui a agonisé pendant cinq heures avant d'être évacué.

23. Ha'aretz, 05/10/2000.

24. Ibid., p. 305.

25. Voir le compte rendu du sondage dans Ha'aretz du 13/10/2000. Ori Nir : « Not by hummus and za'tar alone».

26. Le service de sécurité intérieure.

27. Voir Ha'aretz, 12/10/2000. Amos Arel : « Shin Bet : Israel must integrate its Arabs ».

28. Quarante, très exactement.

29. L'autre nom du Shin Bet, plus volontiers utilisé par la population arabe.

30. Exception faite de sept villes mixtes où une minorité d'Arabes résident dans des quartiers le plus souvent séparés.

31. Par exemple en 2000, les habitants du village de 'Ein Mahel sont venus protester contre des confiscations de terres devant le bureau du Premier ministre à Jérusalem. Le petit attroupement a été quasiment aussitôt dispersé par la police.

32. Elie Rekhess, «The Arabs in Israel and the Intifada », in Robert O. Freedman (ed), The Intifada, its Impact on Israel, the Arab World and the Superpowers, Miami, Florida International University Press, 1991.

33. Voir Le Figaro du 22 mai 1990 où un compte-rendu des événements est fourni.

34. Ibid.

35. Voir en particulier l'article de Michel Warschawski dans le Monde diplomatique de janvier 2000 : «La gauche israélienne retrouve la parole».

36. Voir Ha'aretz du 06/10/2000. Baruch Kra : « Police blame Galilee clashes on the fact that force 'don't existe' in Arab Israeli towns ».

37. Sortes de petites communautés agricoles à l'organisation plus lâche que les kibboutz-s.

38. Ha'aretz 05/10/2000. Israel Harel : « The second battlefront ».

39. Elias Karam dans Kul al-'Arab du 06/10/2000, p. 7.

40. Sawt al-Haqq wa al-Huriyya du 20/10/2000. Mohannad Moustapha : « L'intifada d'al-Aqsa... et la leçon qu'il faut en tirer ».

41. Pour une analyse plus détaillée de ce processus, voir Laurence Louër, « Comment gérer la minorité arabe d'Israël ? Les élections de mai 1999 ", Politique étrangère, nº 2, été 2000 . 
42. Ceci car l'organisation centrale du front est le Parti communiste, longtemps seul parti anti-sioniste qui faisait office de mouvement nationaliste arabe et, de fait, n'avait d'ancrage que dans la population arabe. Son influence chez les Juifs était et est toujours négligeable.

43. L'autre branche du Mouvement islamique a deux députés à la Knesset, élus sur la Liste arabe unie.

44. En particulier le parti ultra-orthodoxe sépharade Shass, dont les services sociaux alternatifs sont en fait financés par les fonds publics du ministère de l'Intérieur, de l'Education ou encore des Religions. A ce sujet voir le livre d'Ilan Greilsammer, Les hommes en noir. Essai sur les partis ultra-orthodoxes, Paris, FNSP, 1991.

45. C'est le cas en particulier à Nahariyya au nord ou à Hadéra non loin de Tel Aviv. 46. Voir par exemple Gilles Kepel pour le cas des mouvements islamistes : Gilles Kepel et Yann Richard (dir.), Intellectuels et militants de l'islam contemporain, Paris, Seuil, 1990.

47. Voir en particulier Susan Olzak, The Dynamics of Ethnic Competition and Conflict, Stanford California, Stanford University Press, 1992.

48. Ainsi que le note Sammy Smooha dans son étude des attitudes réciproques des Juifs et des Arabes. Voir Sammy Smooha, Arabs and Jews in Israel, tome II : Change and Continuity in Mutual Intolerance, Boulder, San Francisco and Oxford, Westview Press, 1992, pp. 236-261.

\section{INDEX}

Mots-clés : conflits, démocratie, exclusion

Index géographique : Israël, Palestine 